\title{
Passive Augmentations in Hearing Protection Technology Circa 2010 including Flat-Attenuation, Passive Level-Dependent, Passive Wave Resonance, Passive Adjustable Attenuation, and Adjustable-Fit Devices: Review of Design, Testing, and Research
}

\author{
John G. Casali \\ Auditory Systems Laboratory, Grado Department of Industrial and Systems Engineering, Virginia Tech, \\ Blacksburg, VA 24061
}

(Received 9 March 2010; accepted 9 June 2010)

\begin{abstract}
Augmentations or enhancements to conventional HPDs, that is, those which attenuate noise strictly through static, passive means, are generally delineated into passive (non-electronic) and active (powered electronic) designs. While powered electronic augmentations are reviewed in Casali ${ }^{1}$ (a parallel paper elsewhere in this issue), passive augmentations are represented by mechanical networks to achieve flat-by-frequency attenuation; level-dependent leakage pathways that house acoustically-variable occluders to yield minimal attenuation during quiet periods but sharply increasing attenuation upon intense noise bursts (such as gunfire); quarter-wave resonance ducts to bolster attenuation of specific frequencies; selectable cartridges or valves that enable passive attenuation to be adjusted for specific exposure needs; and dynamically adjustable-fit devices that provide adjustment features to enable personalized fit to the user as well as some degree of attenuation control. Intended benefits of passive augmented HPDs (akin to those of active devices as well) include (1) more natural hearing for the user, (2) improved speech communications and signal detection, (3) reduced noise-induced annoyance, (4) improved military tactics, stealth maintenance and gunfire protection, and (5) provision of protection that is tailored for the user's needs, noise exposure, and/or job requirements. This paper provides a technical overview of passive augmented HPDs that were available or have been prototyped circa early-2010. In cases where no empirical research results on the passive augmentations and their performance were available in the research literature, this review relied on patents, corporate literature, and/or the author's experience. For certain augmentations, a limited amount of empirical, operational performance research was available and it is covered herein. Finally, in view that at the juncture of this article the United States (U.S.) Environmental Protection Agency (EPA) was in the process of promulgating a comprehensive new federal law to govern the testing and labeling of hearing protectors of various types, those elements of the proposed law that pertain only to specific passive augmentation technologies are mentioned herein, ${ }^{2}$ along with references to relevant standards on hearing protector attenuation testing.
\end{abstract}

\section{INTRODUCTION}

An overview of the state of the technology of hearing protection devices (HPDs) circa 1996 was published by Casali and Berger. ${ }^{3}$ Considering that article is now outdated by 15 years, the purposes of this review are (1) to update the earlier article with coverage of new technology augmentations, specifically on available passive technologies, (but not exhaustively in regard to all manufacturers/models), (2) briefly present the results of relevant research conducted on passive augmented HPDs, and (3) to briefly cover the testing and labeling of passive augmented HPDs as to their attenuation and other performance characteristics under a recently proposed U.S. EPA regulation $^{2}$ that is intended to ultimately supercede the current federal regulation, ${ }^{4}$ which does not accommodate most augmentation technologies, be they passive or active. The testing issue was not addressed in the earlier article, ${ }^{3}$ but it is becoming increasingly important as consumers and safety professionals attempt to select from a variety of augmented HPDs. These HPDs are purported to offer certain hearing and protective advantages, but heretofore they could not be comprehensively tested and properly labeled under the current EPA regulation, for reasons associated with the nonapplicability of the EPA's cited test protocols ${ }^{4}$ to certain dynamic HPD capabilities, as reviewed elsewhere. ${ }^{5}$

\subsection{Definitions Applied}

In this paper and its parallel paper, ${ }^{1}$ the terms "augmentation" and "augmented hearing protector" are intended to refer to any device that does not consist solely of a static passive attenuator, but which includes features involving electronics or dynamic/adjustable passive acoustical impedance elements. Also, the term "active" hearing protector is operationally defined as one which incorporates powered electronics of any type, which are typically powered from a battery source. Herein, the term "active" does not refer exclusively to devices of the active noise reduction type, or of the active sound transmission/restoration type, but instead it encompasses both of these varieties since they include powered electronics design features.

\subsection{Conventional Passive Hearing Protection Devices (HPDs) and Applications Thereof}

The bulk of available HPDs comprise the category of socalled conventional devices. These devices are the subject of 